\title{
Contribution à l'étude des écoulements générés par la houle au travers d'une paroi perforée
}

\author{
Jean-Marc Rousset \\ LMF, UMR 6598 CNRS / Centrale Nantes \\ 1 rue de la Noë - BP 92101 - 44321 NANTES Cedex 03 \\ Ingénieur de recherche - jean-marc.rousset@ec-nantes.fr
}

\section{Résumé}

Les écoulements de la houle à travers une paroi perforée sont complexes et difficiles à étudier. Deux outils, l'un numérique, l'autre expérimental, sont présentés afin de mettre en évidence leurs apports potentiels à l'analyse de ces écoulements ainsi qu'à d'autre domaines du génie côtier

\section{$\underline{\text { Abstract }}$}

Flows generated by water waves passing through a perforated structure are complex and their study are not easy. Two methods, a numerical simulation and an experimental method, are present in order to highlight their potential benefits to these flow analyses and to other coastal engineering fields.

Mots-clés : simulation numérique, modélisation physique, analyse spectrale.

\section{Introduction}

La bibliographie nationale et internationale s'intéressant aux digues à paroi perforée est relativement fournie. Lors de précédentes Journées Nationales de Génie Civil - Génie Côtier, ont été présentées des réalisations d'ouvrages, des modèles numériques et physiques ou bien encore des résultats de mesures sur site. Parallèlement aux problèmes de stabilité et de dimensionnement de l'ouvrage auxquels est confronté le concepteur, le passage de la houle à travers l'écran perforé constitue à lui seul un sujet d'études de mécanique des fluides.

La présente communication vise à présenter deux outils de recherche originaux qui permettent d'appréhender les phénomènes physiques sous des aspects nouveaux et complémentaires. La valorisation de ces méthodes résidera dans leur application à d'autres domaines du génie côtier.

La première partie de l'article présente la modélisation numérique des écoulements à surface libre complexe mettant en oeuvre une méthode dite "Volume Of Fluid". Ce code numérique, issu des travaux de Y. Andrillon ${ }^{1}$, a été validé dans le cadre de problèmes liés à l'hydrodynamique navale. Nous 
présentons ici une première phase de validation concernant l'écoulement de la houle à travers la paroi perforée.

La seconde partie de la communication présente succinctement une méthode récente d'analyse temps-fréquence et son application à des données obtenues lors d'une campagne de vélocimétrie.

\section{L'outil numérique "VOF"}

\section{1. Principe généraux de la modélisation}

Le code numérique employé pour reproduire le fonctionnement hydraulique du caisson Jarlan est un code Navier-Stokes utilisant une méthode de capture d'interface pour représenter la surface libre ${ }^{1,5}$.

La technique de capture d'interface dite VoF revient à modéliser l'écoulement d'un seul fluide dont les caractéristiques physiques, la viscosité et la masse volumique varient au travers d'une zone de transition qui est assimilée à la surface libre. Ces caractéristiques physiques sont déterminées par les relations décrites en (1) qui introduisent la fraction de volume. Cette quantité représente le rapport du volume de fluide 1 sur le volume de l'élément considéré. Elle vérifie une équation de convection (2) qui permet de déterminer l'évolution temporelle et spatiale de la surface libre.

$$
\begin{gathered}
\rho=\frac{c \rho_{1}+(1-c) \rho_{2}}{\rho_{1}} \mu=\frac{c \mu_{1}+(1-c) \mu_{2}}{\mu_{1}} \\
\frac{\partial c}{\partial t}+\vec{\nabla} g(\vec{c})=0
\end{gathered}
$$

Le code de calcul résout un système numérique fortement couplé construit à partir des équations de Navier-Stokes ( 3 et 4). Ces équations sont discrétisées au moyen de la méthode des volumes finis avec une précision d'ordre 2. La discrétisation temporelle employée est de type Euler implicite d'ordre 2. La résolution du système est ensuite effectuée par un solveur de type Bicgstab- $\omega$, couplé à un préconditionement LU.

$$
\begin{gathered}
\int_{V} \frac{\partial \vec{U}}{\partial t} d V+\int_{S}(\vec{U} \otimes \vec{U}) \overrightarrow{d S}+\int_{V} \frac{\vec{\nabla}(P)}{\rho} d V-\frac{1}{\operatorname{Re}} \int_{S} \frac{\mu}{\rho}(\vec{\nabla} \otimes \vec{U}) \overrightarrow{d S}-\int_{V} \frac{\vec{e}}{F r^{2}} d V=0 \\
\int_{S} \vec{U} \overrightarrow{d S}=0
\end{gathered}
$$

Ces principes généraux donnent lieu à une modélisation numérique temporelle développée soit dans une version bi-dimensionnelle (2D verticales dans notre cas) soit dans une version tri-dimensionnelle. Cette dernière, bien qu'exigeant un temps 
de calcul relativement important, devrait apporter l'opportunité d'étudier les écoulements générés par des houles obliques dans un caisson de type Jarlan.

Pour plus de détails sur le code numérique, le lecteur pourra utilement consulter le mémoire de thèse de Andrillon dans lequel il trouvera la description complète de ces simulations et développements numériques, ainsi que la présentation des éléments de validation.

\section{2. Application au caisson à paroi perforée}

L'utilisation du code 2D et son adaptation à une structure maritime constitue une première étape en cours de validation. Afin de permettre la comparaison pertinente des données expérimentales et des résultats de calculs, les conditions expérimentales décrites dans Rousset $^{6}$ ont été reproduites dans la simulation numérique. Les paramètres géométriques de la berme et du caisson à paroi perforée sont donc identiques. De plus la génération de la houle est réalisée au moyen d'une paroi mobile oscillante, à l'image du batteur dans le canal à houle. Seule la distance batteur - structure n'est pas respectée et se trouve plus courte dans le code : numériquement la houle ne se propage que sur quelques longueurs d'onde devant le caisson afin de ne pas trop augmenter les temps de calcul.

La comparaison des mesures expérimentales et des résultats de la modélisation numérique est actuellement conduite à partir d'essais en houle régulière. L'objectif est de confronter ces séries de données pour plusieurs cambrures et longueurs d'onde, en s'intéressant à respectivement les évolutions temporelles :

- de la surface libre devant le caisson et dans la chambre de dissipation ;

- des pressions exercées sur les parois verticales ;

- des écoulements générés par le passage des vagues au travers de la paroi perforée, en particulier les modules des vitesses maximales.

Le code numérique permet en effet la simulation des jets observés lors du passage de la houle à travers la paroi perforée ainsi que le déferlement induit par le déphasage des élévations de la surface libre. Il est à noter que ces phénomènes physiques ne sont pas accessibles au modèle numérique "2D-V" présenté précédemment par Gomi ${ }^{3}$.

\section{3. Comparaison numérique - expérimental}

A titre d'exemple sont présentés les champs des modules des vitesses de part et d'autre de la paroi perforée, pour une hauteur de houle incidente en canal de $0,05 \mathrm{~m}$ de période $1,7 \mathrm{~s}$ (au réel $\mathrm{H}=1,35$ et $\mathrm{T}=8,8 \mathrm{~s}$ ) et une profondeur de $0,30 \mathrm{~m}$. Les vagues se propagent à partir de la gauche du dessin et la paroi arrière de la chambre se trouve à droite.

Les résultats obtenus par la simulation numérique sont présentés lors de deux phases caractéristiques: 1) la phase de remplissage de la chambre et 2) la phase de vidange de cette même chambre (Figure 1). Les mesures obtenues par PIV pour cette dernière phase sont reportées sur la Figure 2. 

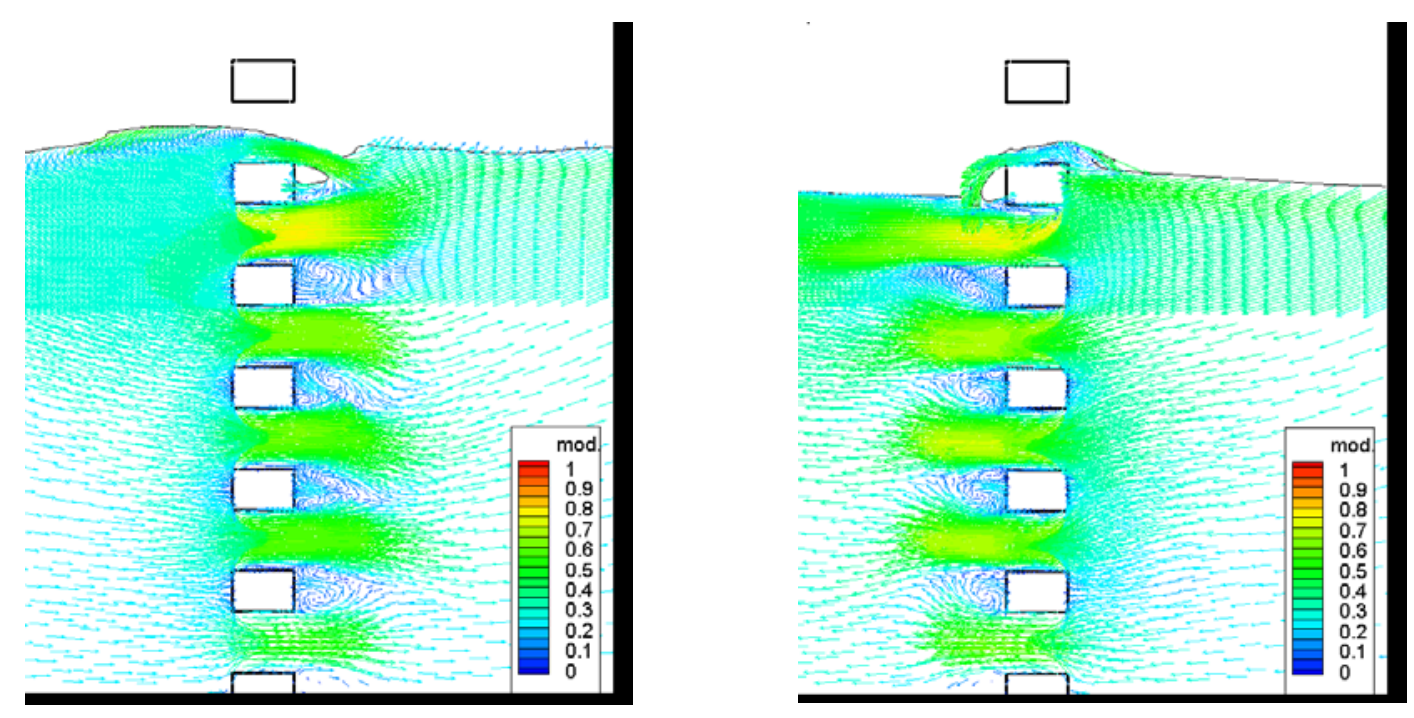

Figure 1: Champs des vitesses obtenus par simulation numérique

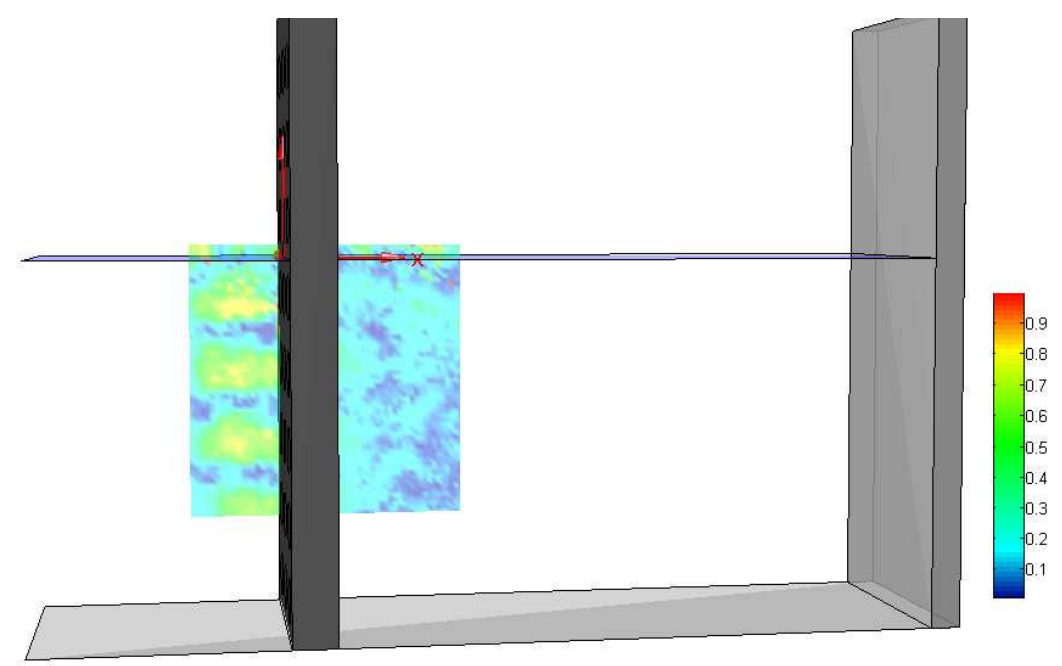

Figure 2: Champs de vitesses obtenus expérimentalement par PIV

La comparaison des champs de vitesses obtenus par le code numérique et expérimentalement par imagerie de particules (PIV) montrent un bon accord. Les ordres de grandeurs des composantes des vitesses ainsi que dimensions caractéristiques des tourbillons sont globalement respectés. L'analyse de la surface libre donne également des résultats en bon accord.

$\mathrm{Si}$ les premières comparaisons entre simulation numérique et expérience sont prometteuses, il reste toutefois des points à améliorer. Une étude systématique de l'influence des paramètres en entrée du code sur la qualité des résultats serait notamment utile pour faciliter la mise en oeuvre de la simulation : domaine de calcul (dimensions du maillage, nombre de cellules, etc), durée de la simulation (nombres de périodes de houle), etc. 


\section{L'analyse temps - fréquence}

\subsection{Analyses par transformées de Fourier et par ondelettes}

D'une manière générale, l'étude des signaux provenant de capteurs, quel que soit leur nature, se fait traditionnellement à l'aide d'une analyse spectrale par transformée de Fourier (TF). Les deux hypothèses qui sont à la base de cette méthode méritent d'être rappelées car elles conditionnent les conclusions qui peuvent être raisonnablement tirées de l'analyse :

- les transformées de Fourier s'appliquent à un système linéaire ;

- le signal, ou l'extrait du signal étudié, est considéré stationnaire ou périodique.

La série temporelle de données revient alors à être regardée comme une somme de sinusoïdes de fréquence données et d'amplitudes constantes, gommant en particulier tous les phénomènes transitoires.

Dans le cas de la propagation d'ondes de gravité comme la houle, en présence ou non d'obstacles, ces deux conditions peuvent ne pas être remplies. Les nonlinéarités sont en effet très importantes tant par leurs amplitudes que par les conséquences qu'elles induisent. Elles représentent par elles-mêmes un intarissable sujet de recherche. La propriété majeure des vagues est également de se propager dans un milieu dispersif liant ainsi longueur d'onde, hauteur, profondeur et direction : il est donc difficile de considérer ce phénomène physique comme stationnaire, même sans déferlement. Selon les critères cités plus-haut, les transformées de Fourier ne seraient pas les meilleurs outils pour analyser et comprendre ces phénomènes physiques, même si leurs définitions mathématiques sont irréprochables et leurs utilisations très courantes.

La théorie des ondelettes a été élaborée pour circonvenir aux restrictions citées auparavant et elle est devenue populaire dans le génie océanique depuis une dizaine d'années. Elle fait partie des méthodes d'analyse temps-fréquence et ses principes sont compatibles avec des signaux présentant des transitoires (données "non-stationnaires"). Il s'agit en effet de corréler le signal original avec une famille de fonctions construite à partir d'une ondelette originale (type Morlet par exemple). Ces ondelettes ont une durée temporelle finie (au contraire des sinusoïdes de l'analyse de Fourier) et une moyenne nulle.

Il reste tout de même un problème lorsqu'il s'agit de traiter des données présentant de nombreuses non-linéarités puisque fondamentalement la décomposition en ondelettes est linéaire. Le choix initial de la famille de fonctions mise en oeuvre est également une question difficile : la décision est prise a priori parmi la douzaine d'ondelettes classiques selon la caractéristique prédominante que l'on souhaite faire ressortir des données (régularité de phénomènes physiques, bruits , etc.). 


\subsection{Analyse par transformées de Hilbert-Huang}

Une autre méthode temps - fréquence est apparue récemment et se base sur les travaux de Norden Huang ${ }^{4}$. Il s'agit de la technique d'analyse par transformées de Hilbert-Huang (ou HHT pour avoir un parallèle avec les FFT). Cette approche est mise en oeuvre dans de nombreux domaines technologiques, parmi lesquels l'aérospatial, la météorologie, le contrôle structurel de machines, son application aux vagues océaniques étant également en plein développement ${ }^{2}$.

La méthode est destinée à analyser les signaux provenant de systèmes nonlinéaires et "non-stationnaires". Elle se base sur une décomposition adaptative (EMD pour Empirical Mode Decomposition), en utilisant les extrema locaux du signal, pour créer des fonctions qui en décrivent les modes intrinsèques. Par hypothèse, chacune de ces fonctions, appelées IMF (Intrinsec Mode Functions), représente une oscillation caractéristique contenue dans le signal original. A la fin du processus de décomposition, le signal $x(t)$ est donc représenté par la somme de $n$ IMF $h(t)$ et d'un résidu que l'on minimise (il correspond en fait à la tendance moyenne du signal original) :

$$
x(t)=\sum_{j=1}^{n} h_{j}(t)+r_{n}(t)
$$

Une IMF peut ainsi permettre de visualiser une modulation en fréquence et / ou en amplitude. Ce point est un des intérêts de la méthode HHT car dans le cas d'un signal modulé de ce type, une analyse par TF donnera un spectre avec des harmoniques dont la réalité physique est sujet à discutions.

Le processus de décomposition en IMF est itératif à partir des courbes enveloppes s'appuyant sur les extrema du signal et en se conformant à deux conditions :

- le nombre d'extrema et de passage par zéro doit être égal (ou au plus différent de 1) au signal initial ;

- à chaque instant, la valeur moyenne de la courbe enveloppe des minima et des maxima doit être nulle.

Cette approche adaptative autorise l'extraction des différents niveaux d'énergie associés à différentes échelles de temps. Elle permet également d'éviter de retrancher le signal moyen du signal, phase préparatoire parfois délicate mais souvent nécessaire à l'analyse par TF.

La décomposition par HHT est également applicable à des signaux dont la fréquence d'acquisition est variable. Cette particularité se rencontre par exemple pour des données de Vélocimétrie Doppler à Laser où la cadence d'acquisition est liée au taux de particules passant dans le volume de mesure. 


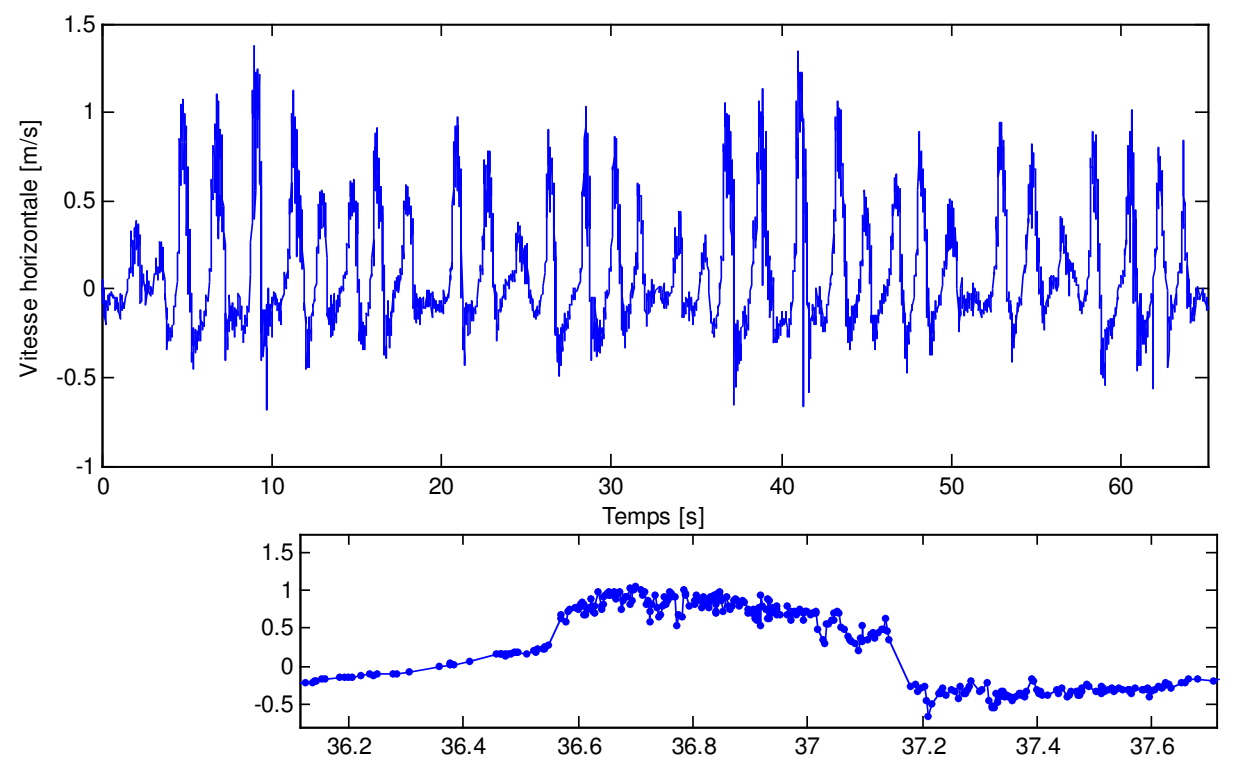

Figure 3: Vitesse horizontale dans un jet

A titre d'exemple, la méthode HHT est appliquée à des données de VDL acquises au cours d'une campagne de mesures en canal à houle, sur une maquette de caisson à paroi perforée. La Figure 3 présente une série temporelle de la vitesse horizontale mesurée au centre d'un jet généré par de la houle irrégulière. La seconde courbe est un extrait de la courbe supérieure : elle montre les bouffées de points lors des extrema de vitesses, phénomène bien connu lié au nombre de particules détectées par l'appareil.
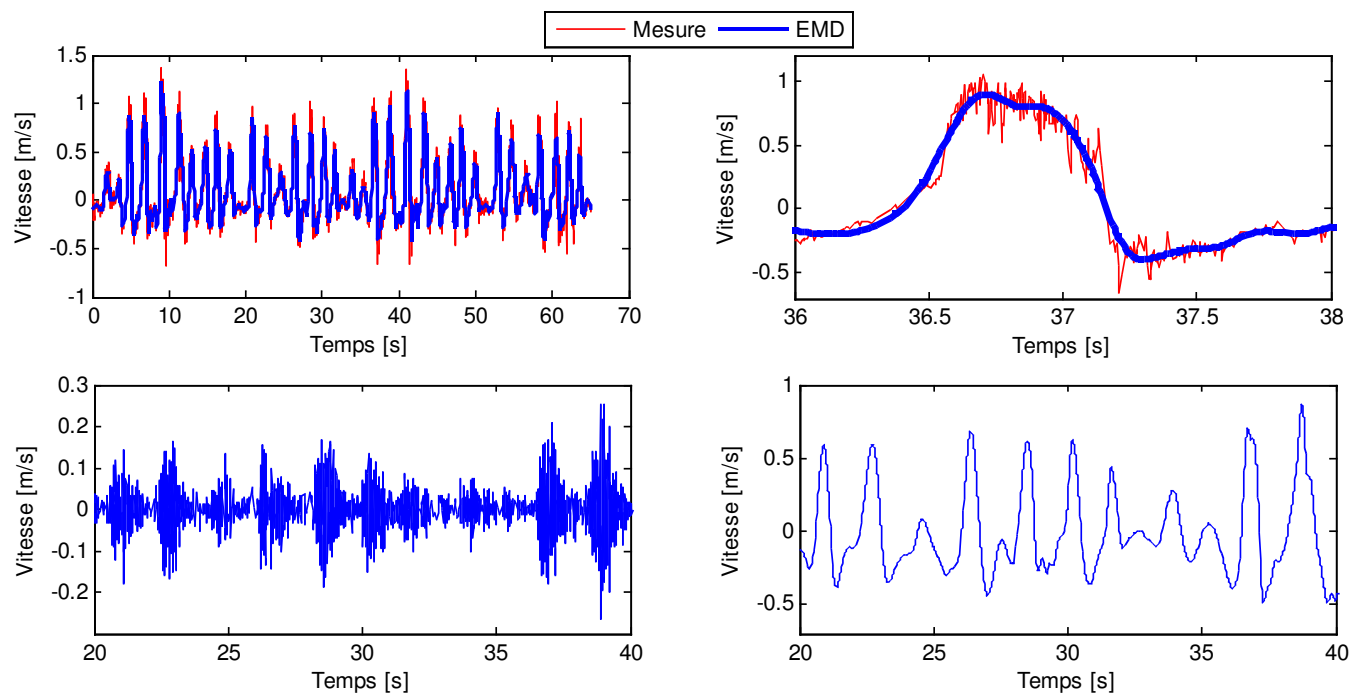

Figure 4: Comparaisons du signal initial avec les modes obtenus par EMD

La décomposition EMD du signal de vitesse permet de séparer des composantes hautes-fréquences et des composantes basses-fréquences sans a priori sur les 
gammes de ces fréquences. Pour ce cas précis, 22 modes différents sont obtenus, certains ayant des amplitudes très faibles, en plus de la tendance moyenne.

Sur la Figure 4 en haut, sont reportées les données initiales et la superposition de 13 modes $\left(n^{\circ} 10\right.$ à 22$)$, pour la totalité de l'enregistrement à gauche et pour un extrait à droite. Ces modes décrivent bien l'évolution globale de la vitesse en filtrant les oscillations hautes fréquences. Sur les courbes du bas de la figure 4 se trouvent deux extraits de 20 secondes pour lesquels sont mis en évidence les composantes hautes-fréquences (superposition des modes 1 à 3 , à gauche) et les oscillations de fréquences plus basses (modes 10 à15, à droite). Ces dernières sont de grandes amplitudes $(-0,5 /+1 \mathrm{~m} / \mathrm{s})$, directement liées aux élévations irrégulières de la surface libre et donc à la houle. Les amplitudes des hautes fréquences provenant des effets de turbulence provoqués par le jet alterné sont 2 à 5 fois plus faibles que les amplitudes basses fréquences. Elles sont néanmoins parfaitement extraites du signal original sans filtrage préalable ni rééchantillonnage, ce qui autorise ainsi la mise en œuvre d'une analyse spectrale sans hypothèse restrictive.

\section{Conclusion}

Dans le cadre d'une étude des écoulements complexes à surface libre appliquée aux digues à paroi perforées, un code numérique VOF est actuellement en cours de validation. Une nouvelle technique d'analyse des données temps-fréquence est également mise en oeuvre et permet de mieux appréhender les phénomènes physiques.

\section{Références}

1 Andrillon Y. (2004). Simulation d'écoulement à surface libre par une méthode de capture d'interface en formulation totalement couplée. Thèse de Doctorat, Ecole Centrale de Nantes.

2 Dätig M., Schlurmann T. (2004). Performance and limitations of the HHT with an application to irregular water waves., Ocean Engineering, vol. 31, pp 1783-1834.

3 Gomi P., Rousset JM., Ropert F. et Sergent P. (2004). Numerical simulation of the Jarlan caissons hydraulic behaviour, Bulletin PIANC - AIPCN, ${ }^{\circ} 117$, pp 15-27.

4 Huang. NE. Et al. (1998). The empirical mode decomposition and the Hilbert spectrum for non-linear and non-stationary time series analysis, Proc. R. Soc. London, Ser. A, vol. 454, pp 903-995.

5 Le Touzé D., Andrillon Y., Ferrant P. et Alessandrini B.(2003). Comparison of VOF and Spectral-potential models on three-dimensional sloshing, $6^{\text {th }}$ Numerical Towing Tank Symposium, Roma (Italy).

6 Rousset JM. and Bruce T. (2004), Study of the flow pattern in a perforated breakwater using PIV measurements, in PIV and Water Waves, Advances in Coastal and Ocean Eng. Vol.9, Grue, Liu \& Pedersen Ed., pp 324-326. 